\title{
Study on Water Conservancy and Hydropower Engineering Risk Analysis
}

\author{
Tianxiao Nan \\ School of Water Conservancy and Hydropower Engineering, Ho Hai University, Nanjing 210000, China
}

\begin{abstract}
The water conservancy and hydropower engineering is built to make full use of the amazing power of water, while there exist variety kinds of risks in the process of the system building and operation. Based on the analysis of the definition and structure of water conservancy and hydropower engineering, this paper summarized the mainly used method in risk analysis and analyzed the risk of water conservancy and hydropower engineering system.
\end{abstract}

KEYWORD: Water conservancy and hydropower engineering; Risk analysis; System risk

\section{INTRODUCTION}

Water is the root of human society. Based on the motive power of the survival and development of human society, the water conservancy and hydropower engineering is built to make full use of the amazing power of water. However, as the water conservancy and hydropower engineering products benefits for our society, it is also can be significantly dangerous when dam-break happened. With the rapid development of social economy, and the accelerating of the process of urbanization, the dam-break risk of the downstream is becoming more and more serious. The center of the safety management of water conservancy and hydropower engineering is shifted to the engineering risk management[1]. No matter what kinds of engineering, there always has the risk of crash which can cause huge loss and severe disasters for human life, economic and environment, figure out how to control those risk is the most important problem of the safety management of water conservancy and hydropower engineering[2]. Therefore, the study on the risk of water conservancy and hydropower engineering has significant theory and practical value.

\section{THE WATER CONSERVANCY AND HYDROPOWER ENGINEERING SYSTEM}

\subsection{The definition of water conservancy and hydropower engineering}

The system of water conservancy and hydropower engineering can be interpreted as the whole process from the planning, survey, design, construction to the operation and management, which includes the main structure of water conservancy and hydropower engineering and the contact interaction between the external environments. The material form of the system is water and water related hydraulic structures, which is the peculiar properties of water conservancy and hydropower engineering system and make it different from other engineering systems. And also because the mainly material form of the system is the water, it is also a subsystem of natural system. It is also a part of our society system which is closely related to national economic development. Therefore, the water conservancy and hydropower engineering system is a complex system both related to nature and society, and it has all features the normal engineering system has.

\subsection{The structure of water conservancy and hydropower engineering system}

According to the definition of water conservancy and hydropower engineering, the system is a hydraulic structures built in a particular location and a particular time. Those different hydraulic structures connected and restricted with each other.

The integrity of the system is the basic of the whole system, the integrity of the water conservancy and hydropower system embodied in the choice of a water conservancy hub location, determine of the shape of dam, the layout of other buildings and the dam construction. The structure and function of the system must be in a benign circulation to fully develop the role and function of the whole system. 
Therefore, the structure and function of the system is very important in the operation of the system.

The structure of the system refers to the form and relationship of each element to be connected, restricted with each other. In the water conservancy and hydropower engineering system, water and different hydraulic structures is the substance elements, the design of water conservancy, the water conservancy and hydropower project bidding, the building construction management and the water conservancy and hydropower engineering construction materials organization, the link between them constitute the system of water resources and hydropower engineering. The function of the water conservancy and hydropower engineering system is the result the interaction of internal and external of the system, which includes flood control, irrigation, power generation, urban and industrial water and other functions.

\subsection{The indefinite factors of the water conservancy and hydropower engineering structure}

1) The uncertainty of load. For hydraulic structure, the load may exist are mainly contains the selfrespect, the water pressure from upstream and downstream, the uplift pressure from the dam foundation and the temperature load. 2) The uncertainty of material parameters. The material parameters include material mechanics parameters and thermal parameters. 3) The uncertainty of geometry size. In the same condition, with different geometric size of the structure, the response of the structure including stress, displacement is not the same. 4) The uncertainty of the initial conditions and boundary conditions. The uncertainty of boundary conditions is derived from the practical problems of complexity, the unpredictability of the change of boundary conditions, and human knowledge limitations 5) The uncertainty of calculation model.

\section{THE RISK ANALYSIS}

\subsection{The definition of risk analysis}

The risk analysis is a comprehensive science gradually developed in recent decades, its originating in the United States, applicate in industry, military, etc. the risk analysis of water conservancy projects also firstly originated in the United States[3].

The risk analysis mainly contains four steps: risk identification, risk estimate, risk avoidance and risk assessment. In the step of risk identification, the first thing is to identify the main risk source may lead to dam failure, then description the model and the logical relationship of various kinds of events may cause dam damage. In the step of risk estimate, the condition probability of each event tree branch and causal relationship between damage forms represented by each branch should be given. In the step of risk avoidance, the engineering and non-engineering measures can reduce the risk of dam failure should be find out. The risk assessment is a decision process, through which can determine what an acceptable level of risk is or what kind of plan to reduce risk and reduce the investment effect is the best.

\subsection{The risk analysis methods}

\subsubsection{The qualitative risk analysis methods}

The qualitative analysis refers to the use of logical methods such as inductive and deductive, analysis and synthesis to research the nature and attribute of things. The qualitative risk analysis methods judge the system risk profile mainly based on the quantitative information like the researcher's knowledge, experience, and the system environment, the trend of the policies and regulations, and special examples. It is usually used on the risk main body which the risk can't be measured specifically. The resources risk and vulnerability of the system can be conveniently analyzed with the qualitative risk analysis. the qualitative methods like the expert experience method, analytic hierarchy process (AHP), matrix analysis and situation analysis, etc. are commonly used in the qualitative risk analysis[4].

\subsubsection{The quantitative risk analysis}

The quantitative analysis refers to the use of quantitative methods and computing tools to research the quantity characteristics, quantity relationship variations, etc. the quantitative risk analysis methods is the study of the relationships and changes of the risk body's quantitative character with the tools of mathematics on the basis of the logic of qualitative analysis. The quantitative methods usually be used in the risk analysis are as follows:

1) The method of mathematical statistics analysis. The extremum statistics and the data statistical analysis methods are mostly used. The extreme statistics is mainly dealing with the maximum and the minimum capacity of a certain sample. As to the data statistical analysis method, the description of statistics and inferential statistics are commonly used tools in risk analysis and uncertainty analysis. The accurate and relevant data parameter is prerequisite of the reliable of risk analysis results. In the water conservancy and hydropower engineering area, the reliability and the resistance factor in risk analysis usually use statistical distribution function to describe.

2) The risk analysis method based on reliability. The commonly used risk estimation methods based on reliability are return period method, the direct integral method, a second-order moment method (FOSM) and its improved algorithm, the point estimation method, the response surface method (RSM) and optimization method, stochastic finite element method (TSFEM), etc. 
3) The risk simulation analysis method. Sometimes there exist relatively complex influence mechanism in the risk analysis which make it difficult to correctly estimate and determine the parameter of the distribution. Therefore, in this situation, using the simulation method for risk analysis is a very effective method. It can be divided into two categories, the deterministic model and probabilistic simulation. The most obvious difference of those two is the inner factor of model, the former is certain while the latter is uncertain. The probabilistic simulation is more suitable for risk analysis; the Monte Carlo method is the most commonly used. The Monte Carlo simulation technique is an effective tool to stochastic simulation, also an effective approach to risk analysis of dam multiple crash model.

4) The fuzzy synthetic evaluation. The fuzzy evaluation analysis can make the fuzzy language variables in the risk analysis quantitatively with membership functions[5]. Because it is difficult to accurately describe the indicators in a specific project or event risk evaluation index system, the fuzzy synthetic evaluation can be used to make synthetic evaluation. Specifically, is to determine the fuzzy comprehensive evaluation index set and gives the comprehensive assessment of the risk level set.

5) The maximum entropy method. The information entropy is basis of the maximum entropy method which is a measure of the uncertainty for random variables in the whole range. In the risk analysis, the information entropy, risk estimate and probability method can be organically linked to establish the maximum entropy risk estimation model. In addition, the grey system theory, Bayesian theory, artificial neural network and genetic algorithm can also be used to make quantitative risk analysis.

\section{THE RISK ANALYSIS OF WATER CONSERVANCY AND HYDROPOWER ENGINEERING}

1) The risk of water conservancy and hydropower engineering system is objective existence because of the difference between people's understanding level and the objective systems. And the development of science and technology also is the result people gradually explored in the process of practice.2) The risk of water conservancy and hydropower engineering system is uncertain. The prediction of the natural river hydrological and flood prediction is conducted on the basis of the statistical law, so as to the analysis of flooding has not yet occurred on river basin is uncertain, which could cause the failure of water conservancy and hydropower engineering system. 3) The effects of water conservancy and hydropower engineering system risk is uncertain. Because of the complexity of the water conservancy and hydropower engineering system, the time and position of the occurrence of the water conservancy and hydropower engineering system risk is uncertain. Sometimes it will encounter difficulties to find the reason in the case of the failure of water conservancy and hydropower engineering system. 4) The risk of water conservancy and hydropower engineering system is predictable. The mathematical statistics and probability methods are often used in risk identify of water conservancy and hydropower engineering system. 5) The risks and benefits of the water conservancy and hydropower engineering is a contradictory unity. The purpose of the construction of water conservancy and hydropower engineering system is to develop the engineering benefit. The efficiency increases, the engineering risk is relatively high, while the efficiency is reduced, it will decrease the risk of project. The risks and benefits of water conservancy and hydropower engineering system is coexisting.

The water conservancy and hydropower engineering system risks have a variety forms of expression. In the different state of the construct of the system, there exist variety kinds of dangerous and risks. Such as in the early exploration stage, if not fully understand the dam foundation and the existence of fracture zone, the risk of dam-break would be happened after the completed of the system which can cause serious harm to people's life and property.

\section{SUMMARY}

According to the risk analysis of water conservancy and hydropower engineering system in the above, it exists many kinds of risks during the construction of the water conservancy and hydropower engineering. Therefore, the construction, operation and management of water conservancy and hydropower engineering system all need to be careful decisionmaking. And on the basis of fully understand the risks, controlling the risk and decreasing the risk damage to the minimum is the main significance of the water conservancy and hydropower engineering risk analysis.

\section{REFERENCES}

[1].Wu, F., Z. Fan, and Z. Tong. Studies of Risk Analysis in Water Resources and Hydropower Engineering Projects Based on Time-variable Effect. In international conference on dam safety management. 2008.

[2].Graham, W.J., A Procedure for Estimating Loss of Life Caused by Dam Failure. Dam Safety Office Us Bureau of Reclamation, 1999. 6(5) (212): p. 262.

[3].Bowles, D.S., L.R. Anderson, and T.F. Glover, The Practice of Dam Safety Risk Assessment and Management: Its Roots, Its Branches, And Its Fruit. It's Roots Its Branches \& Its Fruit Proceedings of Uscold Annual Lecture, 2001. 107(1-3): p. 4757-4764. 
[4].Bao, Y., et al. An improved AHP method in performance assessment. In Intelligent Control and Automation, 2004. WCICA 2004. Fifth World Congress on. 2004.

[5].Zhang, T., et al. Research on the Risk Assessment of Engineering Project Based on Grey Theory and Fuzzy Mathematics. In International Conference on Wireless Communications, NETWORKING and Mobile Computing. 2008. 